-Technology and Methods.

\title{
Placement repeatability of individual oral stent used in radiotherapy of nasopharyngeal carcinoma
}

\author{
Xiao-Qing Liu, 1,2,3 Wei Luo, ${ }^{1,2}$ Shi-Rong Lin ${ }^{1,2}$ and Meng-Zhong Liu ${ }^{1,2}$
}

1. State Key Laboratory of

Oncology in South China,

Guangzhou, Guangdong, 510060,

P. R. China

2. Department of Radiation

Oncology,

Sun Yat-sen University Cancer

Center,

Guangzhou, Guangdong, 510060,

P. R. China

3. Department of Radiation

Oncology,

The First Affiliated Hospital of

Guangdong Pharmaceutical

University,

Guangzhou, Guangdong, 510080,

P. R. China

Correspondence to: Wei Luo

Tel. : 86.20.87343096

Fax : 86.20.87343535

Email: luowei2@mail.sysu.edu .cn

Grant: Sci-Tech Program

Foundation of Guangdong Province

(No. 2007B031505001)

This paper was translated into English from its original publication in Chinese. Translated by:Guangzhou Liheng and Wei Liu

The original Chinese version of this paper is published in: Ai Zheng (Chinese Journal of Cancer) 28(10); http://www.cjesysu.cn/ cn/article.asp?id=15829)

Submitted : 2009-01-06

Revised : 2009-05-29
[Abstract] Background and Objective: Individual oral stent used in nasopharyngeal carcinoma (NPC) patients during radiotherapy can spare the oral mucosa and tongue. This study was to analyze the placement repeatability and feasibility of individual oral stent used in radiotherapy of NPC. Methods: Individual oral stents were made for 17 naive NPC patients, and three lead markers of $2.0 \mathrm{~mm}$ diameter were embedded in each oral stent. The patients wearing individual oral stent were immobilized by thermoplast mask and unerwent CT scan. Before and every week during radiotherapy, orthogonal (antero-posterior and lateral) X-ray films were taken in conventional simulator, respectively. Displacements of leader markers in medio-lateral (M-L), anteroposterior (A-P) and cranio-caudal (C-C) directions were respectively calculated and compared on each patient's X-ray films. At the dose of $40 \mathrm{~Gy}$, a repeated CT scan was done with the same thermoplast mask and positioning markers. The displacements of co-ordinating gravity center of three lead markers and three selected bony structures between two sets of CT images for each patient were calculated and compared by ANOVA. Results: A total of $240 \mathrm{X}$-ray films were taken. The displacements of lead markers were $(0.69 \pm 0.54) \mathrm{mm}$ in $\mathrm{M}-\mathrm{L}$ direction, $(0.49 \pm 0.62) \mathrm{mm}$ in A-P direction, and $(0.56 \pm 0.57) \mathrm{mm}$ in C-C direction, and the overall vector displacement was $(1.20 \pm 0.77) \mathrm{mm}$ (range, 0-4.98 $\mathrm{mm}$ ). In three-dimensional method, the displacements of co-ordinating gravity center of three lead markers were $(0.75 \pm 0.68) \mathrm{mm}$ in $\mathrm{M}-\mathrm{L}$ direction, $(1.25 \pm 1.12) \mathrm{mm}$ in A-P direction, and $(1.06 \pm 0.77) \mathrm{mm}$ in $\mathrm{C}-\mathrm{C}$ direction, and the overall vector displacement was $(2.15 \pm 0.90) \mathrm{mm}$ (range, $1-4.24 \mathrm{~mm}$ ). The displacements between gravity center of three lead markers and three bony structures had no significant difference in $M-L, A-P$, and $C-C$ directions $(P>0$. 05). The overall vector displacements also had no significant difference $(P=0$. 083). Conclusion: The placement repeatability of individual oral dental stent in the mouth is very good during radiotherapy of NPC, and dental stent has a fixed position relationship with bony structures.

Key words: dental stent, nasopharyngeal neoplasm, placement repeatability, setup uncertainty

Radiation-related oral mucositis and taste impairment are the most common adverse effects in patients with head and neck cancer undergoing irradiation. According to literatures, the morbidity and severity of the radiation-induced taste impairment and oral mucositis have close relation with the irradiated dose and volume of tongue and oral mucosa. ${ }^{1-3}$ In the radiotherapy for head and neck tumors, individual dental stent keeps the patients oral cavity open and pushes the tongue downward, so as to artificially increase the distance between the oral mucosa and tongue with 
the target volume, and keep the tongue and most oral mucosa away from high-dose area around; then reduces their total irradiated dose and volume, which helps to reduce the morbidity and severity of radiation-induced mucositis, protect the taste and eventually improve the quality of life for the patients. Qin et al. ${ }^{4}$ reported that, in the conventional radiotherapy for nasopharygeal carcinoma (NPC), the irradiated dose of tongue with dental stent was significantly lower than those without dental stent; patients with dental stent had less tongue injury than control group, and a tendency of decreasing morbidity was seen for mucositis; therefore, individual dental stent is valuable in the radiotherapy for headand-neck tumors. Whether individual dental stent is clinically feasible and worthy of wide application depend on its placement repeatability during radiotherapy.

\section{Materials and Methods}

Case selection. Inclusive criteria were as follows: (1) patients aged of 16-65 years; (2) no other oral disorders, with intact teeth; (3) no major heart, liver or lung function disorders, and could tolerate the entire course of radiotherapy; (4) performance status score $<2$. Exclusive criteria were as follows: those who discontinued the radiotherapy for any other reasons; (2) other oral disorders that interfered with dental stent wearing during radiotherapy.

From January to April 2007, 17 patients with primary NPC who were treated in the first sector of the radiotherapy department in Cancer Center, Sun Yat-sen University were enrolled. Among these patients, 15 were men and two were women. They aged of 24-58 years, with a median of 42 years. All these cases were pathologically confirmed as undifferentiated non-keratinizing carcinoma.

Making of the individual dental stent. After admission, the patients were first sent to the dental department for routine dental cleaning before radiotherapy. Polymethyl methacrylate resin was used as the material of individual dental stent, and a leader marker of $2 \mathrm{~mm}$ in diameter was embeded in both sides and anterior part of the stent. The leader markers on both sides were not on the same horizontal level, and the anterior one was not medially embeded. The individual dental stent included three parts. Fixation piece, which included upper dental incisure and fixing grooves against the lower dental arch, was used to hold the patients mouth open. In addition, the shape of the upper dental incisure and fixing grooves against the lower dental arch was consistent to that of upper and lower teeth, so as to ensure consistent placement of dental stent in oral cavity. Tongue-pusher piece was the main component of the stent, with a thickness of 3-5 $\mathrm{mm}$. Its length was determined based on the body size and the position of the tongue, with its posterior edge extending to the juncture between the middle third and posterior third of the tongue. It played a role by pushing the tongue down to the mouth floor and totally separating the tongue from the soft palate. The tongue-pusher piece was designed to incline slightly downward so that the tongue was pushed forward to prevent the base of the tongue from dropping backward. Medial hole and connecting arch (Fig. 1). When the dental stent was made and placed into the patients oral cavity, examination was performed to see whether the tongue was properly pushed and whether the stent caused any discomfort. If it caused any discomfort, modification was made until the stent was completely fit. In all the patients included, the dental stent was well in place during the radiotherapy. 

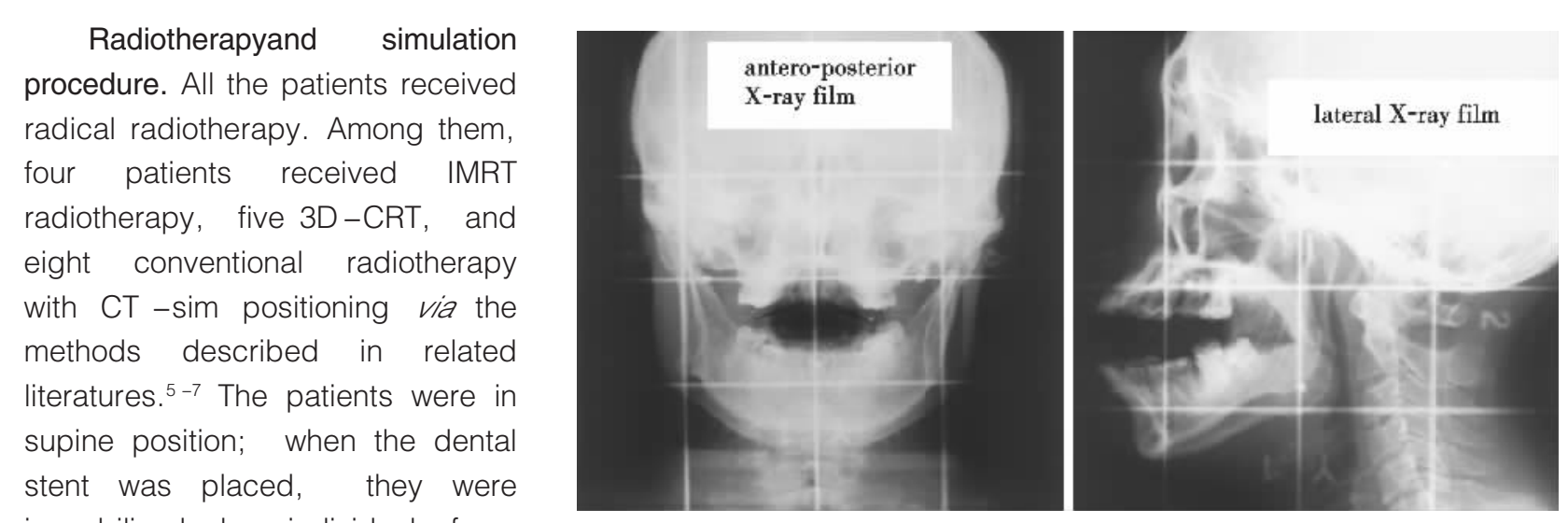

immobilized by individual foam pillow and thermoplastic mask. CT simulation positioning was

Figure 2 The antero-posterior and lateral X-ray films of one patient with conducted by Siemens Plus 4 nasopharyngeal carcinoma

spiral CT system (Siemens), with slice thickness of 3 $\mathrm{mm}$, slice gap of $3 \mathrm{~mm}$ and image matrix of $512 \times$ 512. During scanning, three cross markers were made on the left, medial and right of the mask by a 3-dimensional laser lamp, and then leader beads of $1 \mathrm{~mm}$ in diameter were attached to the cross markers as markers in CT images.

Measurement of the placement repeatability for individual dental stent. Two-dimensional measurement method. When the individual dental stent was in place, the patients were immobilized by mask and underwent CT scanning for treatment plan designing. Before repositioning during radiotherapy, one anteroposterior and one lateral $\mathrm{X}$-ray images of $10 \mathrm{~cm} \times 10$ $\mathrm{cm}$ were taken, centering around the CT markers (antero-posterior and lateral positioning images were shown in Fig. 2). Then, one antero-posterior and one lateral images were taken each week by the concentional $X$-ray simulator under the same conditions during radiotherapy. For each patient, 1416 images were taken, with the $X$-ray image at the first repositioning as reference image, then the images taken each week were compared to it. On lateral image, bony anatomic markers including sphenoid sinus, anterior and posterior clinoid and upper margin of posterior lepospondyli symphysis were used as reference. On antero-posterior image, nasal septum, orbit and maxillary teeth were used as reference. Displacement of three metal markers in $X$, $Y$ and $Z$ axes in relation to these bony anatomic markers were compared on the $X$-ray images. The medio-lateral direction was defined as $X$ axis, and displacement toward the left was recorded as negative value and displacement toward the right as positive value; the antero-posterior direction was defined as $Y$ axis, and displacement forward was recorded as negative value and displacement backward as positive value; the cranio-caudal direction was defined as $Z$ axis, and displacement upward was recorded as negative value and displacement downward as positive value.

Displacements on $Y$ and $Z$ axes were detected on lateral images and displacement on $X$ axis detected on antero-posterior images.

Three-dimensional measurement method. CT images used in the treatment planning for the patients were transferred to the CT-sim workstation, where three metal markers were delineated under bone window; with the automatic center searching function in CT-sim, the coordinates of geometric center of these metal markers was identified. Sphenoid sinus, odontoid process and left maxillary sinus were selected as normal bony markers; with the same method, these markers were delineated and the coordinates of geometric center identified. At the dose of 40 Gy, a repeated CT scan was done with the same thermoplast mask and positioning markers, the body position, immobilization method, scanning variables, and scanning scope were the same as the first CT scan. Again, the structures mentioned above were delineated under the same window width and conditions, and the coordinates of geometric center of three metal markers, as well as sphenoid sinus, odontoid process and left maxillary sinus, were identified by the same method. The identification of bony structures and the measurement of the coordinates were finished by the same physician, in order to avoid individual measurement error between different physicians. Displacement on $X, \quad Y$ and $Z$ axes was obtained by subtracting the first set of 
Table 1 The statistic data of lead marker displacements in two-dimensional method

\begin{tabular}{|c|c|c|c|c|c|c|}
\hline & \multicolumn{4}{|c|}{ Displacement (mm) } & \multirow{2}{*}{$\begin{array}{r}\text { Negative } \\
\text { displacement }(\%)\end{array}$} & \multirow{2}{*}{$\begin{array}{c}\text { Forward } \\
\text { displacement }(\%)\end{array}$} \\
\hline & Mean \pm SD & Minimal & Maximal & Range & & \\
\hline $\mathrm{X}$-axis $(\mathrm{M}-\mathrm{L})$ & $0.69 \pm 0.54$ & 0 & 3.00 & $-3.00 \sim 3.00$ & 46.1 & 46.3 \\
\hline $\mathrm{Y}$-axis $(\mathrm{A}-\mathrm{P})$ & $0.49 \pm 0.62$ & 0 & 3.50 & $-3.50 \sim 3.00$ & 29.4 & 43.7 \\
\hline $\mathrm{Z}$-axis $(\mathrm{C}$ - $\mathrm{C}$ ) & $0.56 \pm 0.57$ & 0 & 4.00 & $-4.00 \sim 3.50$ & 46.6 & 41.4 \\
\hline Vector displacement & $1.20 \pm 0.77$ & 0 & 4.98 & $0.00 \sim 4.98$ & & \\
\hline
\end{tabular}

The displacements of lead markes had been measured for 309 times. M-L: medio-lateral direction; A-P: antero-posterior direction; C-C: craniocaudal direction.

Table 2 The displacement comparison between three lead markers and three bony structures in three-dimensional method

\begin{tabular}{lcccc}
\hline Direction & Gravity center of lead markers $(\mathrm{mm})$ & Gravity center of bony structures $(\mathrm{mm})$ & $F$ value & $P$ value \\
\hline X-axis (M-L) & $0.75 \pm 0.68$ & $0.56 \pm 0.62$ & 0.921 & 0.345 \\
Y-axis (A-P) & $1.25 \pm 1.12$ & $0.56 \pm 0.81$ & 0.144 & 0.707 \\
Z-axis (C-C) & $1.06 \pm 0.77$ & $0.93 \pm 0.92$ & 0.179 & 0.286 \\
Vector displacement & $2.15 \pm 0.90$ & $1.56 \pm 0.95$ & 3.212 & 0.083 \\
\hline
\end{tabular}

All values are presented as mean $\pm \mathrm{SD}$ of 16 measurements. M-L: medio-lateral direction; A-P: antero-posterior direction; C-C: cranio-caudal direction.

coordinates from the second set of coordinates; three-dimensional displacements of geometric center were compared between the three metal markers and the normal bony markers, so as to reveal whether there was difference. Definitions of $X, \quad Y$ and $Z$ axes were the same as in two-dimensional measurement.

Statistical methods. The data was analyzed using SPSS software version 12.0; descriptive statistical analyzing methods were used to describe the displacement of metal markers in 3 -dimensional direction by 2 -dimensional method; analysis of variance was used to reveal the difference between the displacements of geometric centers of the three markers and the three normal bony structures, with two-sided test. A $P$ value $<0.05$ was considered as significant.

\section{Results}

Two-dimentional method. Of the 17 patients, five had taken 16 images, nine had 14 images, two had 12 images and one had 10 images, with a total of 240 images (120 antero-posterior images and 120 lateral images). $\quad X$-ray images taken at the first repositioning were used as reference image for the measurement of displacement of the metal markers. The absolute displacements on $X, Y$ and $Z$ axes were shown in Table 1. The 3 -dimensional vector displacement was $(1.2 \pm 0.77) \mathrm{mm}$ (range, $0-4.98$ $\mathrm{mm}$ ). The displacement was $>3 \mathrm{~mm}$ in $3.4 \%$ of the patients, and was $>2 \mathrm{~mm}$ in $6.9 \%$ of the patients (Table 1).

Three-dimensional method. Due to absent repeated CT scanning, one patient was excluded. Thus data were collected from a total of 16 patients. The absolute displacement and 3-dimensional vector displacement on $X, Y$ and $Z$ axes of geometric center of the three metal markers and the three normal bony markers were shown in Table 2. Analysis of variance showed no significant difference in displacement between them $(P>0.05)$.

Comparison of the displacements of metal markers at the dose of 40 Gy by 2 -dimensional method and by 3-dimensional method

Analysis was conducted on the displacement of metal markers at the dose of 40 Gy in 2-dimensional method. The displacements on $X, Y$ and $Z$ axes were $(0.84 \pm 0.55) \mathrm{mm},(0.91 \pm 0.74) \mathrm{mm}$ and $(0.92 \pm$ $0.81) \mathrm{mm}$, respectively, and 3 -dimensional vector displacement was $(1.70 \pm 1.01) \mathrm{mm}$. These results were compared with the displacements of metal markers in 3 -dimensional method by analysis of variance, which showed no significant difference $(F=$ 1.525, $\quad P=0.222 ; \quad F=1.807, \quad P=0.184 ; \quad F=0.368, \quad P=$ 0.546; $F=3.140, F=0.082)$. 


\section{Discussion}

In the radiotherapy for head and neck tumors, individual dental stent is commonly used in the clinical practice. In China, cork or mouthpiece are also used to separate the tongue and part of the oral mucosa from target volume, as to reduce radiation dose of the tongue and oral mucosa. Reitemeier et al. ${ }^{8}$ tried to use 4 different types of substances including gold compound, pure titanium, amalgam and an artificially synthetic material as the materials of dental stent to avoid the directly radiation to the tongue and oral mucosa, and revealed substantial efficacy. However, all these materials could scatter a certain amount of radiation and thus increase the radiation dose of the surrounding tissue. Moreover, cork or mouthpiece can not be individualized, therefore they may be not comfortable to wear and with its poor placement repeatability and the possibility of respiratory obstruction, it was hard to be a real tongue pusher.

We selected polymethyl methacrylate resin (formula: $\mathrm{CH}_{2} \mathrm{C}\left(\mathrm{CH}_{3}\right) \mathrm{COOCH}_{3} ; \mathrm{MW}$ : 100.12; relative density was 1 in relation to water), a common material in dental department, as the material for individual dental stent. During radiotherapy, a dental stent made of this material has little influence on the dose of the surrounding tissue. Due to the non-toxic nature of the material, the dental stent is not irritative to oral tissue and is comfort to wear. Once shaped, the stent maintains its volume and shape. Primary dosage studies and clinical studies suggested it played a role by protecting oral mucosa and tongue, ${ }^{4}$ and should be more widely used in clinical practice. However, before that, it should be determined whether its placement is repeatable during radiotherapy.

We measured the displacement of three metal markers of the dental stent on $X, Y$ and $Z$ axes by 2dimensional method and revealed small displacements, with a mean displacement of less than $1 \mathrm{~mm}$ and a mean 3 -dimensional vector displacement of less than $1.2 \mathrm{~mm}$. Which suggested that, by using bony structures as reference markers and comparing the $X$-ray images taken each week with reference image taken at the first repositioning, the placement of dental stent was well repeatable. Analysis of variance was used to compare the displacements on $X, \quad Y$ and $Z$ axes as well as the 3- dimensional vector displacements of geometric center of the three metal markers and the three normal bony structures by 3 -dimensional method, and revealed no significant difference. It suggested that the placement of dental stent was well repeatable, and that the dental stent had an almost fixed relationship with the bony markers and the displacement of dental stent was representative of that of the bony markers, which indicated the dental stent was clinically feasible. Lin et al. ${ }^{9}$ conducted CT scanning every week to detect the set-up error in patients with NPC; the results suggested that displacements of bony markers on $X, Y$ and $Z$ axes were $(0.89 \pm 0.69)$ $\mathrm{mm}, \quad(0.82 \pm 0.79) \mathrm{mm}$, and $(0.95 \pm 1.24) \mathrm{mm}$, respectively, and the 3 -dimensional vector displacement of geometric center was averagely 1.87 $\mathrm{mm}$, which were similar to the displacements of normal bony structures as measured by 3dimensional method in our study.

However, in 2-dimensional method, the maximal overall 3-dimensional vector displacement of three metal markers was $4.98 \mathrm{~mm}$, and the overall 3dimensional vector displacement was $>2 \mathrm{~mm}$ in $6.9 \%$ of the patients, and was $>3 \mathrm{~mm}$ in $3.4 \%$ of the patients; the maximal displacement on $X, Y$ and $Z$ axes could be 3.5-4 mm, which indicated poor placement repeatability of dental stent in some patients. The potential reasons for large displacement are as follows: (1) the fixing groove against lower dental arch was shallow, leading to poor biting; the dental stent was thus moved backward, resulting in large displacement in antero-posterior direction; (2) the patient put on the stent hastily, therefore the upper dental incisure was not well biting; (3) the patient became emaciated after the radiotherapy, and thus the mask became looser. Hence, it is necessary to deepen the fixing groove against lower dental arch; meanwhile, the patient should be reminded to ensure good biting of the dental stent before radiotherapy, so as to decrease the displacement.

The displacement of metal markers detected by 3 -dimensional method was larger than that by 2 dimensional method, but when compared to the displacement of metal markers measured by 2 dimensional method at the dose of $40 \mathrm{~Gy}$, the difference was not significant. The reasons for a larger displacement of metal markers measured by 3 -dimensional method than that by 2 -dimensional method might be as follows: (1) The dental stent had 
a fixed relationship with the bony structures, but not with the soft tissue. The 3-dimensional measurement was only repeated once at the dose of about 40 Gy, when most patients are emaciated to a certain degree. Getting wasting facial and cervical soft tissue changed the relation between CT markers and bony anatomic structures, therefore displacement was also detected for normal bony structures. Such issue also could be found in 2-dimensional method, and the displacement increased as radiotherapy proceeded. But due to multiple 2-dimensional measurements, the mean value of displacement were corresponding reduced. (2) The CT markers in repeated CT scanning were re-made with the same method as in the first CT scan, errors in making positioning markers might lead to inaccurate identification of marker coordinates. (3) In CT scanning, the spatial resolution on $Z$ axis was poor (slice thickness was 3 $\mathrm{mm}$ and slice gap was $3 \mathrm{~mm}$ ), which might increase errors in the displacement on $Z$ axis.

The measurement was conducted by $X$-ray convention simulator, not on treatment conditions, that was the shortcoming of 2-dimensional measurement. In addition, we only performed one measurement per week; a small number of measurements might result in a smaller displacements than the actual one. Lin et al. ${ }^{10}$ measured the set-up errors of bony markers on $\mathrm{X}$ ray simulator and on treatment machine, and found significant differences between the two results. Therefore, to improve the accuracy of the measurement, it was necessary to increase the number of measurements and to measure the actual displacement on accelerator. On the other hand, it was preferable to repeat CT scanning every week as well, so as to improve the accuracy of measurement, and to provide more accurate data for the modification and the widespread use of dental stent.

In conclusion, both 2-dimensional and 3-dimensional measurement results suggested good placement repeatability of dental stent in the radiotherapy for NPC, where the stent had an almost fix relationship with the bony structures. 3D-CRT and IMRT render the 3-dimensional distribution of high dose conformal on the shape of the tumor, and thereby provide better protection for surrounding vital organs and also make the possibility of increasing the radiation dose in tumor,then subsequent improving the tumor control probability. But the variations of patient position and radiation field in each radiotherapy course may lead to dose uncertainty in the margin of target volume. If the margin between CTV and PTV is underestimated, tumor control probability (TCP) may be decreased, whereas an overestimated margin may result in increased normal tissue complication probability (NTCP). Hence, studies on the placement repeatability in radiotherapy for tumor have important implications. Numerous ways can be used to measure set-up errors, such as bony markers, parapharygeally implanted metal markers, mouthpiece or denture embeded with three metal balls. Pan et al. ${ }^{11,12}$ and Oita et al. ${ }^{13}$ used denture or mouthpiece embeded with metal markers to detect the set -up error in head-and-neck tumors. However, before using this method, it is necessary to establish a fix position relation between metal markers and tumor or bony markers, so that the displacement of metal markers can representive the set-up errors. Therefore, we should first demonstrate the placement repeatability of denture or mouthpiece is very good. The results of our study suggested excellent placement repeatability for the dental stent; the dental stent had an almost fix relationship with the bony structures, and its displacement was generally representative of that of the bony structures.Meanwhile, anterior and posterior clinoid and sphenoid sinus are considered immobile organs, thus their displacements are representative of that of the tumors. As a result, using three metal markers implanted into the dental stent as internal markers, is a good way to detect set-up error. In addition, IGRT is currently widely used in radiotherapy for tumors, so as to decrease the influence of organ swaying and set-up error on the therapy, and to lessen PTV margin and thus to offer better protection for normal tissue.As compared with bony markers, metal markers produce clearer projection and are easier to match. With furtherly improved placement repeatability of the dental stent, the placement relation between target volume and the metal markers on dental stent can be monitored on real-time during radiotherapy. By comparing these real-time images to the reference image, instantaneous modification on patient position can be made automatically to reduce random error and increase treatment accuracy and thereby provide better protection for normal tissue.

In the radiotherapy for NPC, the placement of individual dental stent is well repeatable, and has an almost fix relationship with the bony structures. Therefore, individual dental stent is feasible in 
radiotherapy and worthy of widespread use. In addition, it can be used to detect set-up error and could be attempted in IGRT.

\section{References}

[1] Mantiti G, Manfrida S, Cellini F, et al. Impact of dose and volume on radiation-induced mucositis [J]. Rays, 2005,30(2): $137-144$.

[2] Fernando IN, Patel T, Billingham L, et al. The effect of head and neck irradiation on taste dysfunction: a prospective study [J]. J Clin Oncol, 1995,7(3):173-178.

[3] Mossman KL. Quantitative radiation doseresponse relation-ships for normal tissues in man. I. Gustatory tissue response during photon and neutron radiotherapy [J]. Radiat Res, 1982,91(2): 265-274.

[4] Qin WJ, Luo W, Lin SR, et al. Sparing normal oral tissues with individual dental in radiotherapy for primary nasopharyngeal carcinoma patients [J]. Ai Zheng, 2007,26(3):285-289. [in Chinese]

[5] Zhao C, Lu TX, Han F, et al. Clinical study of 139 nasopharyngeal carcinoma patients of intensity modulated radiation therapy $[\mathrm{J}]$. Zhong Hua Fang She Zhong Liu Xue Za Zhi, 2006,15(1):1-6. [in Chinese]

[6] Luo W, Deng XW, Lu TX. Dosimetric evaluation for three dimensional conformal, conventional, and traditional radiotherapy plans for patients with early nasopharyngeal carcinoma [J]. Ai Zheng, 2004,23(5):605-608. [in Chinese]
[7] Lu LX, Zhao C, Han F, et al. Initial study of the clinical target volume delineation for radiotherapy of nasopharyngeal carcinoma $[\mathrm{J}]$. Zhong Hua Fang She Zhong Liu Xue Za Zhi, 2005,14(2):81-85. [in Chinese]

[8] Reitemeier G, Schmidt A, Schaal W, et al. Evaluation of a device for attenuation of electron release from dental restorations in a therapeutic radiation field $[\mathrm{J}]$. J Prosthet Dent, 2002,87(3):323-327.

[9] Lin CG, Li GW, Lin LW, et al. Set-up uncertainties with radiation therapy for nasopharyngeal carcinoma [J]. Zhong Hua Fang She Zhong Liu Xue Za Zhi, 2006,15 (6):504-507. [in Chinese]

[10 ] Lin CG, Lin LW, Qi ZY, et al. Analysis of factors influencing the accuracy inradiotherapy positioning for nasopharyngeal cancer patients [J]. Zhong Liu Xue Za Zhi, 2005,11(5):321333. [in Chinese]

[11] Pan JJ, Zheng BH, Zhang Y, et al. Measurement of setup errors in conformal radiotherapy for nasopharyngeal carcinoma $[\mathrm{J}]$. Ai Zheng, 2006,25(1):115-118. [in Chinese]

[12] Zheng BH, Pan JJ, Xu LY, et al. Measurement of setup errors in conformal radiotherapy for nasopharyngeal carcinoma by embedded markers in oral occlusal pad [J]. Zhong Hua Fang She Zhong Liu Xue Za Zhi, 2005,14(3):218-221. [in Chinese]

[13] Oita M, Ohmori K, Obinata K, et al. Uncertainty in treatment of head-and-neck tumors by use of intraoral mouthpiece and embedded fiducials [J]. Int J Radiat Oncol Biol Phys, 2006,64 (5):1581-1588. 